\title{
Une clinique possible avec les malades d'Alzheimer*1
}

\author{
Cristina Fontela*2 \\ Thierry Darnaud*3
}

\begin{abstract}
Dans cet article les auteurs montrent qu'une psychothérapie d'inspiration psychanalytique est possible avec des sujets institutionnalisés présentant une démence du type Alzheimer. Elle se justifie d'autant plus que le sujet a subi des pertes relationnelles et d'objets libidinalement investis. Le clinicien tente de s'adapter à un psychisme en proie aux troubles cognitifs où les mouvements transféro-contretransférentiels sont massifs et ont un rôle particulier dans l'économie psychique du sujet. L'accompagnement des angoisses massives de la personne âgée autorisé par la position clinique du psychothérapeute lui a permis de réinvestir la relation à l'autre. Ce travail psychothérapique s'est soldé par une réassurance et par une diminution des troubles psycho-comportementaux d'un sujet éprouvant un phénomène de déréalisation en lien avec des troubles neurologiques liés à l'âge.
\end{abstract}

Mots clés: Alzheimer, Psychothérapie, transfert, contre-transfert

${ }^{* 1}$ Article rédigé à partir du Mémoire de Master 2 Professionnel (Madame Lumière: la question du psychisme dans la maladie d'Alzheimer) présenté à l'U.F.R. de Psychologie sous la direction de M. Thierry Darnaud, soutenue en septembre 2011 à l'Université de Toulouse - Jean Jaurès.

*2,3 Université Toulouse - Jean Jaurès (Toulouse, France) 


\section{Introduction}

Vieillir est un processus inéluctable. C'est une étape incontournable de la vie et tout un chacun devra y faire face le moment venu. Or, le vieillissement est un processus inévitable, et il est généralement vécu comme une épreuve qui traverse le corps avec ses multiples pertes et déficits. Face à ce constat de la réalité présente, le sujet en processus de vieillissement ressent les effets du temps qui passe et refuse cette évidence, car le vieillissement n'existe pas dans l'atemporalité de l'inconscient (Link, 2007).

Pendant longtemps les sujets âgés ne se voyaient pas proposer une psychothérapie. Freud (1898/1976) considérait en son temps que la psychanalyse ne s'appliquait pas aux personnes âgées du fait d'un excès de matériel psychique exigeant beaucoup de temps pour être analysé. De plus, les individus âgés seraient caractérisés par une viscosité de la libido, cette expression faisant référence aux difficultés que la personne âgée rencontrerait pour changer ses investissements libidinaux. Talpin (2011) objecte deux arguments à la thèse de Freud. D'abord un sujet jeune pourrait présenter un excès de matériel psychique et mobiliser les résistances pour éviter d'aborder des sujets essentiels pour lui. Ensuite, les sujets âgés, contrairement à ce que l'on pourrait supposer, iraient à l'essentiel car motivés par le sentiment d'urgence du temps qui reste à vivre.

L'intérêt de ce travail se situe dans les dénouements du transfert et du contretransfert pendant la prise en charge d'une malade d'Alzheimer, et le rôle qu'ont joué ces deux processus dans son mouvement psychique vers la réassurance, un retour au Soi, et un ancrage dans le réel. Cet article s'appuie sur un cas clinique pour démontrer l'importance et la pertinence de l'accompagnement clinique des sujets présentant un syndrome démentiel. En permettant un réinvestissement narcissique pour le sujet, en s'appuyant sur sa subjectivité et sa capacité d'association, qui certes ne s'expriment plus comme avant car moins perceptible mais qui n'en restent pas moins encore intactes, la prise en charge psychothérapique apporte un bénéfice certain pour la diminution des angoisses générées par les pertes de repères spatio-temporels. 


\section{ARTIGO}

Ferrey \& Le Gouès (2008) considèrent que la cure psychique, ainsi que la psychothérapie psychanalytique, sont identiques pour l'adulte et la personne âgée. L'analyse du contretransfert peut se faire tout en respectant le setting, et en mettant en place des aménagements pour ce faire. Zeppellini Jr. (2008) décrit avec beaucoup de sensibilité ce nouveau setting avec le sujet en processus démentiel:

Dans la clinique du dément le psychothérapeute est confronté à une particularité, à savoir le temps dans le transfert. L'atemporalité de l'inconscient, et par conséquent des symptômes, oblige le clinicien à transiter par les temps des souvenirs, du refoulé, du trauma - qui font surface dans la relation patient/psychothérapeute. Dans le cas particulier des démences, le temps présent n'est plus un élément qui intègre le setting de la rencontre clinique. Les murs de l'institution, les jours de la semaine, les saisons, les horaires des séances - tout élément qui est de l'ordre de l'actuel, du présent devient de moins en moins un objet d'investissement. (p. 47)

Le travail clinique présuppose d'accueillir la parole du dément, de l'accompagner jusqu'au bout avec toute la difficulté psychique que soulève la démence. Tout cela n'est pas très simple pour le psychothérapeute puisque c'est à partir de ses propres chaînes associatives que celui-ci doit trouver du sens dans la discontinuité du discours du dément (Quaderi, 2013). Ensuite, dans ce mouvement relationnel, nous devons prendre en compte les mouvements contretransférentiels du clinicien. En effet, même en ayant aménagé la configuration du setting, le clinicien doit toujours faire face à cette nouvelle relation que le patient entretient avec le temps, car pour le dément, le setting devient une référence de temps, du temps à être partagé pendant la séance, ce qui peut être pour lui source d'angoisse (Zeppellini Jr., 2008). Dans ce nouveau contexte du setting, les paramètres de la cure sont réévalués en fonction des changements observés dans l'économie psychique du sujet et, si besoin, le psychothérapeute est amené à changer son plan d'accompagnement psychologique, et à aider la personne âgée jusqu'au bout dans ce processus de vie (Le Gouès \& Ferrey, 2008). Le changement que la démence provoque au niveau psychique et comportemental exige une prise en charge psychologique adaptée, qui prend en compte l'expérience que le sujet âgé fait de lui-même avec l'arrivée de la maladie. Ploton (2001) avance que le placement en institution suite à une hospitalisation peut provoquer des troubles du comportement qui vont se rajouter à la problématique du sujet. Le vécu d'abandon et l'impossibilité de mettre des mots sur des émotions, vont jouer un rôle important dans la tendance des malades à réagir sur un mode comportemental. Un travail d'accompagnement relationnel éviterait l'apparition de troubles psychocomportementaux qui sont alors le seul mode possible d'expression de la souffrance des malades car, comme nous le savons, le dément communique l'inadéquation de l'environnement dans lequel 
il évolue par une augmentation de l'intensité des troubles du comportement (Quaderi, 2013). Ces troubles s'inscrivent dans le registre abandonnique; les liens qui fonctionnent le mieux seront les liens affectifs qu'on parviendra à tisser avec le malade lors du travail psychothérapeutique (Ploton, 2011).

Nous allons présenter la rencontre entre la psychologue et Mme L., une résidente de 85 ans hébergée dans une Unité Protégée d'un Centre Hospitalier. Mme L est rentrée à l'unité Alzheimer en décembre 2010, suite à une hospitalisation pour un état confusionnel et des fugues. Nous avons fait le choix d'une position clinique pour accompagner la souffrance et les angoisses massives de Mme L., à savoir son processus de déréalisation et ses pertes de lien. Nous verrons comment cette position psychothérapique a pu lui permettre de réinvestir sa relation à l'autre et de retrouver une assurance dans sa perception du réel.

\section{Récit du cas clinique}

La rencontre avec Mme L. a été la conséquence d'une demande d'une aide soignante de l'unité protégée où elle résidait depuis trois mois. L'aide soignante expliquait que Mme L. demandait incessamment de rentrer chez elle. La résidente ne savait pas où elle vivait mais son désir de partir s'actualisait chaque jour. Lors de notre premier entretien dans l'infirmerie, Mme L. nous a pris pour la patronne. Ce signifiant nous indiquait qu'elle actualisait le passé dans le présent et agissait comme si elle était encore à son travail à l'usine, ou chez les gens pour faire le ménage. Mme L. est née en Italie en 1927. Fille d'un père hongrois et d'une mère autrichienne, elle a vécu avec ses parents pendant quelques temps en Italie. Puis, en raison des exactions de l'Etat Fasciste italien, la famille a fui définitivement l'Italie pour s'installer en France. Après l'obtention de son Certificat d'Etudes Primaires, Mme L. arrête ses études. Elle travaille alors comme boulangère, puis dans une biscotterie, ensuite comme femme de ménage et garde d'enfants. Elle épouse un jeune homme, qui travaille à l'usine avec elle, et parle de lui avec tendresse. Il meurt dans un accident du travail. Elle épouse son deuxième mari vers l'âge de 30 ans. Pendant plusieurs années elle essaye d'avoir des enfants, sans succès. Finalement, après avoir suivi une assistance médicale à la procréation, elle tombe enceinte vers l'âge de 39 ans et donne naissance à une fille. Mme L. n'évoque pas beaucoup son deuxième mari. Elle parle de lui comme un pauvre chien perdu sans collier. Elle dit que sa belle-mère aimait tous les hommes sauf son fils. Ceci explique, selon elle, le fait que son mari était si malade et qu'il ait été interné en hôpital psychiatrique pendant plus de douze ans. Mme L. est très fière de sa fille et aime beaucoup ses deux petits-enfants. 


\section{ARTIGO}

\section{Le premier contact: une rencontre singulière}

Notre première rencontre s'est déroulée dans l'infirmerie de l'unité où Mme L. est conduite par l'aide soignant. Elle dit en me regardant droit dans les yeux qu'elle veut bien travailler avec moi [...] qu'elle peut tout faire, du repassage, faire les lits... Mais qu'il faut qu'entre nous tout soit au clair car elle n'aime pas les histoires. Après m'avoir longuement parlé, elle me demande de ne raconter à personne ce qu'elle me dit. Cette précaution de Mme L. nous rappelle le sentiment de persécution caractéristique de la symptomatologie de la maladie d'Alzheimer. Elle rappelle aussi l'expression d'une angoisse signal d'alarme (Freud, 1926/1975), une stratégie du Moi pour faire face à la situation de danger appréhendée par le sujet en début de démence. Nous la rassurons quant à notre discrétion et nous lui proposons de la rencontrer ultérieurement. Elle s'assure que notre parole sera tenue et nous confirme son accord pour une prochaine rencontre.

Proposer une psychothérapie au sujet âgé présentant des troubles cognitifs renvoie à la reconnaissance de sa souffrance et de sa douleur psychique face à l'angoisse et à un vide qui s'installe au fur et à mesure que le processus évolue (Montani, 1994). C'est affirmer que le sujet reste un être de subjectivité et d'intersubjectivité dans son rapport à l'autre, même s'il n'a plus tous ces moyens pour le signifier. Or, les pertes cognitives mettent à mal le tissage des liens actuels et effacent graduellement les liens anciens avec les êtres familiers. Le vide psychique qui est ressenti de façon consciente par le sujet provoque chez lui une déstabilisation majeure dans l'appareil psychique. Le Moi est ébranlé et une surexcitation pulsionnelle peut alors prendre le dessus. C'est donc dans un contexte d'angoisses et de déréalisation que nous avons fait connaissance avec Mme L. trois mois après son entrée dans l'unité protégée.

\section{Un objet transitionnel: le chat Billy}

$\mathrm{Au}$ cours des premiers entretiens qui ont lieu dans sa chambre, Mme L. nous montre les photos de son chat Billy et parle beaucoup de lui. Elle prend les photos, elle les touche, les regarde avec un sourire. Le temps que Mme L. consacre à parler de son chat représente le lien avec sa vie antérieure et permet l'expression de l'affection. En parlant de lui, elle parle de la voisine qui l'a gardé, de sa fille, de ses petits enfants et d'une vie qui est restée derrière elle.

La complexité du processus démentiel avec les pertes cognitives, l'atteinte narcissique, la perte d'autonomie et l'institutionnalisation, mettent le sujet dans un sentiment de grande vulnérabilité et de souffrance. Selon Ploton (2011) le vécu 
d'abandon éprouvé par le dément l'amènerait dans le besoin d'une présence, voire d'une présence maternelle lors d'épisodes confusionnels. Le vécu d'abandon et ce besoin d'une présence pourrait bien s'apparenter à la déprivation affective qu'expérimentent les enfants lors d'un placement (Winnicott, 2010). Les objets transitionnels aideraient à compenser la perte de l'environnement familial et, dans le cas des personnes âgées désorientées, la perte de leur domicile, de leur environnement familier. Le chat pour Mme L pourrait bien représenter son univers transitionnel ou de transition. Le chat, qui était auparavant un objet investi affectivement, prend toute sa place dans la transition entre l'ancien et le nouveau. Comme le souligne Winnicott, dans l'espace de l'analyse, n'importe quel objet peut servir d'objet transitionnel.

\section{Le quotidien de Madame $L$.}

A son arrivée dans l'institution, Mme L. se trouvait désorientée. Au fur et à mesure qu'elle retrouvait la marche, elle s'est mise à chercher une porte qui lui permettrait de sortir de l'institution. L'opposition bienveillante des soignants a amplifié chez elle le sentiment de persécution. Les observations que nous avons pu faire pendant les moments d'échanges entre la résidente et les soignants nous ont permis d'entrevoir son double rapport avec la réalité. Dans une démarche thérapeutique, les aides soignantes proposaient à Mme L. de réaliser quelques tâches ménagères. A son tour Mme L. demandait aux soignantes de la laisser faire les lits. Or, dans le processus de déréalisation, il y a une actualisation du passé dans le présent. Mme L. se croit encore dans la vie active, ce qui semble lui donner du sens et une position à sa nouvelle vie dans l'unité. Elle se comporte dans l'unité comme si elle était encore au travail et cherche donc activement à aider. Mme L. établit un clivage en séparant d'un côté les aides-soignants, et de l'autre côté les résidents. Dans un registre identificatoire, les soignants sont gentils car ouvrières comme elle, tandis que les résidents sont des gens qui n'aiment pas travailler.

$\mathrm{Au}$ prise avec des angoisses générées par ses pertes de repères Mme L. vit son lieu de vie comme très insécurisant et l'étrangeté de l'endroit facilite certainement ses productions délirantes. Dans l'ouvrage Inhibition, symptôme et angoisse Freud (1926/1975), fait la distinction entre l'angoisse automatique provenant du ça, de l'inconscient dans lequel il n'y a pas de correspondance avec l'environnement externe du sujet, de l'angoisse signal d'alarme, engendrée par le moi et perçue comme venant de l'extérieur, ce qui témoigne d'un certain degré d'organisation psychique. Chez Mme L. nous retrouvons ces deux types d'angoisse. L'angoisse automatique, se donne à voir dans les moments de 


\section{ARTIGO}

délire ayant pour thème la guerre de 1914-1918; quand elle parle de la mort des membres de sa famille et du traumatisme de savoir que des enfants ont été massacrés. L'angoisse signal d'alarme, quant à elle, est liée à son environnement immédiat. Dans l'unité il y a une résidente qui déambule dans les chambres et emporte des objets avec elle. Mme L. exprime sa colère contre cette personne en déclarant que: dans cette maison il y a des fous qui rentrent chez moi et qui prennent tout. Ainsi, au moment de sortir de sa chambre, elle met son chat en bibelot dans son sac à main et l'emporte avec elle. Dans l'angoisse signal d'alarme, le sujet met en œuvre des opérations défensives qui témoignent d'une activité de symbolisation. Ainsi a-t-elle insisté auprès des soignants pour avoir une clé pour fermer sa porte. Une fois qu'on lui a donné la clé ce comportement de suspicion et de crainte d'être volée s'est arrêté.

Un autre mécanisme psychique inconscient qui nous intéresse ici est celui de clivage. Il serait mis en place pour répondre à un besoin de maîtriser l'angoisse par deux réactions simultanées et opposées, l'une cherchant la satisfaction, l'autre tenant compte de la réalité frustrante. Le clivage est généralement réversible, temporaire, et il est présent dès les débuts de la vie psychique. Il permet l'organisation des émotions, des sensations et des pensées, condition préalable à tout processus d'intégration et de socialisation. L'unité protégée n'est plus alors vécue comme une maison de fous mais comme une maison de retraite.

Pour tenter de comprendre le déclin du psychisme, Péruchon (2011) rapproche le concept d'involution à celui de psycholyse (Ferrey \& Le Gouès, 2008). Dans le phénomène de psycholyse, la personne perdrait plus ou moins vite toutes les capacités complexes de symbolisation, d'abstraction, de mémorisation, pour arriver jusqu'au niveau du vide psychique. Il faut noter cependant que malgré l'apparence de vide psychique que renvoie le dément, il est toujours possible de retrouver chez lui de la subjectivité à condition que le mouvement contretransférentiel du psychanalyste ne bloque pas son écoute du langage du sujet pensé alors sans esprit (dé-ment).

Mme L. se trouve quelque part dans ce processus d'involution. Elle est encore capable de réaliser le tissage symbolique, l'investissement d'objet malgré les défaillances mnésiques et l'affaiblissement de la barrière InconscientPréconscient. Cet affaiblissement de la barrière Inconscient-Préconscient peut être entrevu dans un épisode de délire au cours duquel elle transporte une petite fille d'une chambre à l'autre, ainsi qu'à l'occasion d'un épisode de délire mystique. Des raptus psychotiques peuvent se développer dans la démence de type Alzheimer et prendre parfois une ampleur telle que le mode psychotique peut sembler être devenu le fonctionnement psychique de la personne (Ferrey \& Le Gouès, 2008). D'après Péruchon (2011) au début des troubles mnésiques l'entrée psychique dans la démence est organisée par la personnalité antérieure du sujet. 
Selon sa capacité de sublimation et le soutien reçu de son entourage, le dément parvient à se maintenir à distance de la dépression et des pertes narcissiques. En revenant sur les premiers jours de Mme L. dans l'Unité, nous remarquerons qu'elle a subi non seulement la perte des objets investis libidinalement, mais également de son environnement relationnel. Ainsi, proposer une psychothérapie à la personne en processus démentiel signifie la construction d'une écoute attentive, libidinalement affectueuse, ce qui reviendrait à offrir au sujet âgé la possibilité d'une satisfaction soutenue par l'étayage dans le présent (Zeppellini Jr., 2008).

\section{Quand l'angoisse prend le pas dans le psychisme}

Dès le début de la prise en charge nous avons remarqué que Mme L., malgré sa souffrance psychique, était capable d'investissement libidinal. Nous avons choisi de conduire les entretiens dans sa chambre afin de rester dans un lieu tranquille et de pouvoir parler sans être constamment interrompus. Sa chambre était le setting privilégié pour la majorité des entretiens. A chacun de nos entretiens, elle plongeait dans ses souvenirs. Elle parlait de ses parents, de l'histoire familiale, de son mari, de sa fille.

Les premiers entretiens ont été difficiles à suivre car les récits se présentaient sans logique apparente dans le temps et dans l'espace. Les personnages de son histoire se mélangeaient de façon confuse. Cela nous obligeait à une tentative de reconstruction de sens au moment de chaque récit. Il fallait adapter l'écoute pour ne pas être envahi par la fatigue et maitriser nos propres résistances. Au début de la prise en charge, la question du transfert n'était pas claire car Mme L. nous voyait, à ce moment là, comme une ouvrière. Cette projection identificatoire ne laissait pas de place pour déceler la/les sources à l'origine de son angoisse, de sa déréalisation et la grande confusion dans ses récits ne laissait pas entrevoir le mouvement transférentiel.

Il est devenu plus aisé d'identifier clairement le transfert de Mme L. à l'occasion de ses délires et hallucinations au sujet d'une petite fille. Ce matin là, une infirmière nous informe que Mme L. était très en colère la nuit précédente, qu'elle avait beaucoup déambulé dans les chambres à la recherche d'une petite fille. Nous allons la voir dans sa chambre. Elle est très énervée. Elle dit qu'elle a installé dans une chambre une petite fille de 10-11 ans et que celle-ci a ensuite disparu. Elle accuse les infirmières de l'avoir envoyée ailleurs. Notre question de départ s'adressait à la représentation de cette enfant dans le délire de Mme L. et à la possibilité du retour du refoulé. Que représente cette petite fille pour 


\section{ARTIGO}

Mme L.? Quels sont les éléments de la réalité de l'institution qui rejoindraient la réalité subjective de la résidente et déclencheraient son épisode de délire? Mme L. croit que les soignantes veuillent supprimer l'enfant car elles n'aiment pas les catholiques, ces protestantes. Quand on lui demande qui est cette enfant elle ne veut pas le dire car c'est un secret. D'après elle, les parents de l'enfant ont été tués; elle a donc recueilli cette pauvre orpheline. Pour l'instant nous l'écoutons et nous essayons de trouver du sens à cette construction hallucinatoire. Nous cherchons des mots clé pour nous aider à réfléchir, associer. Il nous vient à l'esprit le mot perte. Perte d'objets libidinalement investis. Lesquels? Nous essayons d'avoir plus d'informations sur l'enfant. Avec l'hypothèse qu'en réalité il s'agit d'une déréalisation liée à l'angoisse de perte d'objet, ou d'une angoisse d'abandon, nous prenons le risque d'avancer en lui posant encore des questions, malgré sa colère. Il est comment cet enfant? Elle nous dit que c'est une petite fille de 9-10 ans. C'est une enfant née prématurée. Dans son histoire personnelle, Mme L. a eu une sœur jumelle morte à la naissance. Ici, il nous semble qu'elle met à jour, par hallucination mnésique, un scénario ancien, c'est-à-dire le scénario de la naissance et du décès de sa sœur d'après les explications données par sa famille. Nous revenons à notre hypothèse selon laquelle la déréalisation de Mme L. serait liée à l'angoisse de perte d'objet ou à l'angoisse d'abandon. Nous lui demandons de décrire la petite fille qui a disparu. Elle dit: Elle est un peu comme vous, un peu algérienne. Nous croyons comprendre que la création hallucinatoire de Mme L. est en relation avec le transfert et son investissement dans la relation avec la psychologue. Cette hypothèse est-t-elle plausible? Nous lui demandons pourquoi elle nous a cherché. Elle nous répond que c'était pour vous prévenir. Je me suis dit que ce pauvre bougre qui ne fait du mal à personne, qui protège les autres. Ces sont des fascistes. En poursuivant son discours, elle dit qu'elle a entendu, ou qu'elle a rêvé, que ma voiture était dans le fleuve et qu'elle était inquiète pour moi. Nous essayons de rapprocher les faits pour comprendre l'enchainement des événements qui pourraient être à l'origine de la déstabilisation psychique. Effectivement, le début de cette déstabilisation coïncide avec notre absence la veille. Mme L. se montre inquiète. Elle demande aux soignants le numéro de téléphone de la psychologue pour l'appeler et savoir pourquoi elle n'est pas venue. Mme L. s'imagine qu'on veut faire du mal à la psychologue. Il y a de l'angoisse, à la fois primaire et secondaire, car il nous semble que l'absence de la psychologue, qui peut être traduite par l'angoisse d'abandon, a provoqué cette situation. Nous pensons avoir une réponse à travers son rêve sur la voiture de la psychologue dans le fleuve. Mme L. marquait à peu près régulièrement les jours de la semaine prévus pour les rendez-vous sur un calendrier que nous lui avions proposé. Ne voyant pas la psychologue venir le jour prévu pour l'entretien, des angoisses fortes ont émergé. 
Jusqu'alors nous n'avions pas réalisé qu'un transfert d'une telle ampleur était à l'œuvre. La psychologue était bel et bien un objet affectivement investi. Nous supposons que c'est son côté protecteur envers la psychologue qui s'exprime par la voix du rêve, donc de l'inconscient; mais également dans le réel, parce que Mme L. se met en action et cherche les soignantes; elle leur demande le numéro de téléphone de la psychologue. La question qui nous semble alors légitime est de savoir si ce mélange réel-déréel ouvre la voie vers un possible ancrage dans le réel? La relation thérapeutique étayerait-elle un possible retour progressif au réel? Avant de tenter de répondre à ces questions, il ne faut pas oublier que les tempêtes transférentielles sont propres aux psychothérapies du troisième âge (Ferrey \& Le Gouès, 2008). L'appareil psychique du sujet âgé est toujours prêt à un investissement sur la personne qui l'accompagne. Des affects très puissants font éruptions, des affects d'amour ou de haine massifs. Le psychothérapeute doit être avisé de ce transfert. Le travail de répression des affects est d'autant plus coûteux à réaliser que le Moi du sujet âgé s'affaiblit. Mme L. a investi la psychologue par des affects positifs: elle est devenue ouvrière comme elle, puis sa fille.

\section{Sa volonté de partir avec la psychologue}

Quelques jours après l'arrêt des hallucinations et des délires avec l'enfant imaginaire, nous apprenons par les soignants que Mme L. annonçait qu'elle allait partir avec la psychologue. Pour rassurer les soignants, nous avons répondu qu'il ne fallait pas s'inquiéter et que nous discuterions de ce sujet avec Mme L. Le lendemain, à peine arrivée dans l'unité, les aides-soignantes se précipitent vers nous pour nous dire, pour la troisième fois, que Mme L. veut partir avec la psychologue. Cette information a provoqué en nous une suspension de la pensée, une sidération, et le contre-transfert s'est érigé immédiatement. Nous avons décidé d'aborder avec Mme L. le fait que les aides-soignantes nous disent qu'elle veut partir avec nous. Très tranquillement, elle déclare que oui, qu'elle peut partir avec nous, qu'elle allait prendre sa retraite et qu'elle pouvait arrêter de travailler dans la maison et aller travailler chez nous. Nous lui expliquons qu'elle ne pourra pas partir avec nous. Face à son insistance, nous nous entendons répondre: je ne sais pas encore où je vais aller. Ce à quoi Mme L. rétorque: vous allez à T.? Je pars avec vous. Nous insistons en lui disant: je n'ai pas une maison de retraite. J'ai juste mon chez moi. Et puis, je pourrais aller ailleurs, à l'étranger même. Mme L. répond: ah, prendre l'avion et tout? Nous: oui. Mme L.: Dans ce cas, je reste. Nous voyons bien dans cet échange combien la psychologue s'est 


\section{ARTIGO}

laissée prendre dans le contre-transfert sans en avoir conscience. La situation prend une tournure irréelle. Alors que nous ne pensions pas avoir une affiliation réelle avec Mme L., il apparait évident que nous avions tissé avec elle un lien non conscient. Il semble possible d'avancer l'hypothèse que la psychologue avait pris symboliquement un rôle filial. Comme le souligne Soares (2005) "le grand écart d'âge entre le psychothérapeute et la personne âgée peut perturber la création d'empathie et mettre le psychothérapeute dans un rapport de responsabilité vis-à-vis de la personne" (p. 161). Le transfert massif peut alors provoquer chez le psychothérapeute une grande angoisse. Celle-ci se sent envahi par la résidente, ce qui a provoqué un contre-transfert contre-productif pour la psychologue. Il ne s'agissait pas d'une cure dans le sens psychanalytique classique, mais d'une psychothérapie d'inspiration clinique qui essaye de s'adapter à un psychisme en proie à la démence. Est-ce l'investissement affectif de Mme L. qui a pu la faire basculer de la position de se sentir mère à une position de sujet? Rappelonsnous que pendant la psychothérapie, Mme L. a pris la psychologue pour une ouvrière, puis pour sa fille, pour finalement la reconnaitre comme la psychologue qui l'accompagne. Tous ces mouvements psychiques apportaient son lot de résistances chez la psychologue, car la question principale qui se posait concernait sa neutralité et la bonne distance à laquelle se maintenir. Le souci portait toujours sur les issues possibles dès lors que l'on se laisse prendre par les constructions du patient.

\section{Le pari d'une psychothérapie pour les déments}

En mettant en perspective l'évolution de Mme L. dans l'unité Alzheimer nous pouvons dégager quelques aspects qui nous semblent importants. D'abord sa capacité à faire face, à exister dans le monde par et à travers le symptôme sa déréalisation - et sa capacité à communiquer. Le dément communique à travers ses troubles du comportement, à travers ses demandes à répétition, sa déambulation (Quaderi, 2013; Ploton, 2011) quelque chose qui le trouble et l'angoisse. Cette communication a aussi une fonction relationnelle car elle interpelle l'autre qui ne peut pas rester indifférent.

Nous avons rencontré Mme L. dans une grande détresse psychique qui l'a poussé à fonctionner de façon clivée, en processus déréalisé dans un environnement que lui semblait hostile. L'environnement étant le principal vecteur possible de contact avec un monde qu'il cherche encore à retrouver, le malade souffre de l'absence du familier, de l'absence d'objets libidinalement 
investis, car il ne retrouve que de l'étranger partout.

Chez Mme L. une solution du Moi pour exister dans son nouveau lieu de vie, vécu comme angoissant, serait de fonctionner sur le mode psychotique. Le clivage et le déni envers les aides-soignantes et les autres résidents ont opéré pendant un certain temps avant qu'elle retrouve l'apaisement. Ces mécanismes de défense fonctionnent comme des organisateurs de la vie psychique et permettent l'organisation des émotions, des sensations et des pensées (Soares, 2005). Vers la fin de la psychothérapie Mme L. est moins déréalisée et l'unité protégée n'est plus vécue comme une maison de fous mais comme une maison de retraite.

L'identification projective tout au long de la prise en charge semble avoir permis une restructuration psychique. Identifier la psychologue à la patronne au cours de la première rencontre, et ensuite à une ouvrière fait lien dans l'histoire de Mme L. puisque elle-même a été ouvrière. Bion (1983) développe l'idée que "l'identification projective est un mécanisme structurant qui autorise le sujet à retrouver sa capacité à penser" (p. 120). Mme L. se sent proche de l'ouvrièrepsychologue par ce mécanisme là.

La notion de transfert étant centrale dans la cure psychanalytique et clairement présente dans toutes les psychothérapies, nous le repérons aussi dans 456 l'accompagnement de l'ensemble des patients présentant un processus démentiel auquel nous proposons un suivi psychologique. Cependant, le setting avec ses patients demande quelques aménagements et exige une attention redoublée dans le repérage des processus psychiques chez le patient et chez le clinicien. Il faut anticiper les fréquentes tempêtes transférentielles de ces sujets. L'appareil pulsionnel du sujet âgé étant toujours prêt à un investissement sur la personne qui l'accompagne, le thérapeute doit être avisé de ce possible transfert massif. Nous supposons que ce qui a permis à Mme L. de se réapproprier elle-même a été la prise en compte de sa singularité, la possibilité qui lui est donnée de raconter son histoire, de laisser parler sa souffrance malgré la répétition, malgré la difficulté qu'éprouve le clinicien à accompagner son récit. Si l'histoire de vie de Mme L. est effectivement émaillée de moments difficiles: les douze années d'hospitalisation de son mari en psychiatrie, la mémoire profonde et traumatisante transmise par la famille du vécu de la guerre, c'est bien la richesse de ce matériel qui a permis à notre accompagnement d'être psychothérapique pour elle. Comme le souligne Zeppellini Jr. (2008) “ce qui importe dans la psychothérapie, c'est la construction d'une écoute attentive, libidinalement affectueuse, qui offre à la personne âgée la possibilité d'une satisfaction étayée par un lien actuel"' (p. 112).

Accompagner un dément "est un engagement éthique qui nous pousse à considérer les déments autrement que sous le seul aspect comportemental et à nous tourner vers eux, à les recevoir, à les écouter, à les soutenir" (Quaderi, 2013, 


\section{ARTIGO}

p. 13). C'est reconnaitre l'existence d'un sujet dans la démence, et reconnaitre qu'il est un être de subjectivité avec une vie psychique (Quaderi, 2008; Messy, 1992) à qui nous pouvons et devrons toujours proposer une psychothérapie.

Remerciement: à Madame Sandrine Thonnerieux pour l'encadrement institutionnel.

\section{Références}

Bion, W.P. (1983). Réflexion faite. Paris: PUF.

Ferrey, G., Le Gouès, G. (2008). Psychopathologie du sujet âgé. Paris: Masson.

Freud, S. (1975). Inhibition, symptôme et angoisse. Paris: PUF, 1975. (Travail original publié en 1926).

Freud, S. (1976). A sexualidade na etiologia da neurose. Obras completas, ESB, vol. XII. Rio de Janeiro: Imago. (Travail original publié en 1898).

Link, P. (2007). Rencontre avec le sujet dans la maladie d'Alzheimer. Pari d'un ultime transfert. Le Journal des Psychologues, 250, 37-41.

MESSY, J. (1992). La personne âgée n'existe pas: une approche psychanalytique de la vieillesse. Paris: Rivage.

Montani, C. (1994). La maladie d'Alzheimer: quand la Psyché s'égare. Paris: l'Harmattan.

Péruchon, M. (2011). La maladie d'Alzheimer: entre psychosomatique et neuropsychanalyse. Paris: Hermann.

Ploton, L. (2001); La personne âgée. Lyon: Chronique Sociale.

Ploton, L. (2011). Ce que nous enseignent les malades d'Alzheimer. Lyon: Chronique Sociale.

Quaderi, A. (2013). Approche non médicamenteuse de la Maladie d'Alzheimer. Bruxelles: De Boeck.

Quaderi, A. (2008). A psicanálise sob o risco da demência. Psic. Clin., 20(2), 185-198.

Soares, F.M.P. (2005). Envelhescência e pathos: o lugar simbólico das psicopatologias na velhice. Dissertação de Mestrado em Psicologia Clinica. Pontifícia Universidade Católica de São Paulo - PUC-SP.

Talpin, J.M. (2011). Penser le vieillissement entre pathologie et création. Etudes, 4154-2, 43-53.

Winnicott, D.W. (2010). Les objets transitionnels. Paris: Payot.

Zeppelini Jr., J.C. (2008). Demências: Identidades que adoecem. Considerações sobre os aspectos psicopatológicos do envelhecimento. Dissertação de Mestrado em Psicologia Clinica. Pontifícia Universidade Católica de São Paulo - PUC-SP. 


\section{Resumés}

(Uma clínica possível com os doentes de Alzheimer)

Neste artigo, os autores mostram que uma psicoterapia de inspiração psicanalítica é possivel com sujeitos institucionalizados e que apresentam uma demência do tipo Alzheimer. Ela se justifica ainda mais pelo fato de o sujeito ter sofrido perdas relacionais e de objetos libidinalmente investidos. O clínico procura se adaptar a um psiquismo com perturbações cognitivas, no qual os movimentos de transferência e contratransferência são maciços e têm um papel importante para a economia psíquica do sujeito. O acompanhamento das angústias maciças da pessoa idosa, autorizado pela posição clínica do psicoterapeuta, permitiu ao sujeito reinvestir a relação com o outro. Esse acompanhamento psicoterapêutico resultou em um sentimento de segurança e uma diminuição dos distúrbios psicocomportamentais de um sujeito que vivenciava um fenômeno de desrealização em razão dos distúrbios neurológicos ligados à idade.

Palavras-chave: Alzheimer, psicoterapia, transferência, contratransferência

(A possible clinical psychotherapy aimed at patients suffering from Alzheimer's disease)

This article shows that psychotherapy inspired by psychoanalysis may be applied to institutionalized patients suffering from Alzheimer's disease. That kind of psychotherapy is all the more justified by the fact that patients are affected by the loss of their relationships and libidinally invested objects. The clinician has to adapt to a psyche suffering from cognitive disorders where the motions of transference and countertransference are massive and play an important role in the psychic economy of patients. Following up on the massive distress of the elderly, which was made possible due to the clinical status of the psychotherapist, allowed patients to reinvest the relationship with the other. This psychotherapeutic follow-up resulted in a sensation of safety and in the decrease of psycho-behavioral disorders of patients who experience a phenomenon of de realization caused by the neurological disorders related to their age.

Key-words: Alzheimer, psychotherapy, transference, countertransference

(Posible psicoterapia para pacientes con Alzheimer)

En el presente estudio, los autores explican que es posible aplicar un tipo de psicoterapia inspirada en el psicoanálisis a sujetos institucionalizados que sufren la demencia del Alzheimer. Esta psicoterapia se justifica aún más porque en muchos casos el sujeto ha perdido parientes y objetos sexualmente invertidos. Los ensayos clínicos tratan de adaptar una psiquis que ha sido sometida a trastornos cognitivos en los cuales los movimientos de transferencia y de contratransferencia son masivos y 


\section{ARTIGO}

tienen un papel importante en la economía psíquica del sujeto. El seguimiento de las angustias masivas de la persona anciana autorizado por la posición clínica del psicoterapeuta permitió que el sujeto reinvirtiese la relación con el otro. Este seguimiento psicoterapéutico tuvo como resultado un sentimiento de seguridad y una disminución de los trastornos emocionales y de comportamiento que derivaba en un fenómeno de irrealidad sin motivo de los trastornos neurológicos vinculados a la edad.

Palabras claves: Alzheimer, psicoterapia, transferencia, contratransferencia

(Eine mögliche klinische Begleitung von Alzheimer Patienten)

Der vorliegende Artikel zeigt, dass eine psychoanalytisch orientierte Therapie durchaus bei institutionalisierten Alzheimerpatienten angewendet werden kann. Dieser Ansatz rechtfertigt sich insbesondere durch die von diesen Patienten erlebten Verluste von Beziehungen und libidinös besetzter Objekte. Der Kliniker muss dabei eine Psyche berücksichtigen, die an kognitiven Störungen leidet, bei der die Übertragung und Gegenübertragung stark ausgeprägt sind, was das psychische Gleichgewicht der Betreffenden stark beeinflusst. Die Behandlung starker Ängste von älteren Menschen und die Förderung ihrer Beziehungsfähigkeit werden durch den klinischen Status des Psychotherapeuten ermöglicht. Diese psychotherapeutische Arbeit leistet einen wichtigen Beitrag zur Stärkung des Sicherheitsgefühls und zur Verminderung der psychischen und Verhaltensstörungen von älteren Menschen, die aufgrund altersbedingter neurologischer Erkrankungen dem Phänomen der Derealisation ausgesetzt sind.

Schlüsselwörter: Alzheimer, Psychotherapie, Übertragung, Gegenübertragung

\section{(对于阿尔茨海默氏症患者的一个可行临床治疗)}

在这篇文章中，作者展示了对于拥有阿尔茨海默氏症类型的痴呆症患者可 通过心理分析学获得治疗。其原因主要是因为此症患者失去人际关系及具有性 欲寄托的物体。治疗者必须适应患者的知识障碍, 其移情及反移情巨大而对患 者的心里能量有很大的作用。对于老年人的焦虑的疏通可通过心理治疗师的治 疗地位使得患者再建立人与人之间的关系。此种治疗导致患者感到安全而减轻 此类患者脱离现实的困扰。

关键词：老年痴呆症，心理疗法，移情，反移情

Citação/Citation: Fontella, C., Darnaud, T. (2015, setembro). Une clinique possible avec les malades d'Alzheimer. Revista Latinoamericana de Psicopatologia Fundamental, 18(3), 445-460. 
Editores do artigo/Editors: Prof. Dr. Manoel Tosta Berlinck e Profa. Dra. Sonia Leite

Recebido/Received: 7.6.2014/ 6.7.2014 Aceito/Accepted: 25.6.2014 / 6.25.2014

Copyright: (C) 2009 Associação Universitária de Pesquisa em Psicopatologia Fundamental/ University Association for Research in Fundamental Psychopathology. Este é um artigo de livre acesso, que permite uso irrestrito, distribuição e reprodução em qualquer meio, desde que o autor e a fonte sejam citados / This is an open-access article, which permits unrestricted use, distribution, and reproduction in any medium, provided the original authors and sources are credited.

Financiamento/Funding: A autora declara não ter sido financiada ou apoiada / The author has no support or funding to report.

Conflito de interesses/Conflict of interest: A autora declara que não há conflito de interesses / The author has no conflict of interest to declare.

\section{Cristina Fontella}

Docteur en psychologie et psychologue clinicienne.

34, rue Claude de Forbin Apt. 37

31400 Toulouse, France

e-mail: cristinafontella@gmail.com

\section{Thierry Darnaud}

Docteur en psychologie, thérapeute familial et maître de conférences a l'Université de Toulouse Jean Jaurès.

5, allées Antonio Machado

31058 Toulouse Cedex 9, France

e-mail: darnaud@univ-tlse2.fr 